INTERNATIONAL DESIGN CONFERENCE - DESIGN 2018

https://doi.org/10.21278/idc.2018.0178

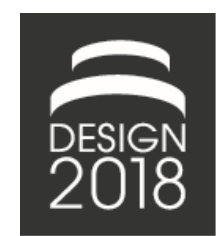

\title{
COMPREHENDING THE DESIGNER'S SKETCH \& IMPLICATIONS FOR COMMUNICATION
}

\author{
J. A. Self
}

\begin{abstract}
Communication between stakeholders during design ideation is important due to growing product complexity. We examine how sketches implicate stakeholder response. Eye-tracking data revealed designers attended to visual representation, while managers focused on diagrammatic elements. Cognitive workload suggested designers adopt a holistic frame-of-reference in comprehending design through sketches. Managers and engineers understand sketches as concrete solutions, leading to increased cognitive workload. Implication for communicated through sketch representation are finally discussed.
\end{abstract}

Keywords: design cognition, new product development, conceptual design

\section{Introduction}

Co-design (Sanders and Stappers, 2008) between various disciplines provides opportunity to make a positive contribution to the design of more appropriate products. This is because the ability of individual stakeholders to apply wider and more diverse knowledge and skills to the new product development process provides the potential for the application of various perspectives and skill-sets in the identification and development of more appropriate solutions (Ulrich and Eppinger, 2012). In the Codesign approach, knowledge is not only shared and applied during evaluation of conceptual solution ideas, as in user-centred design (Holtzblatt et al., 2005), but also contributes to the conceptual proposition of potential solution ideas through a participator design approach.

However, the inclusion of different stakeholders during new product development, particularly at the conceptual stage, risks miscommunication resulting in increased potential for failed concept selection (Nikander et al., 2014). This is because the decisions made in the concept development phase largely determine the quality, cost, and desirability of the end product, as decisions can often only be compensated through high redesign costs and increased development time during later phases (Ulrich and Eppinger, 2012). Effective communication during product development is therefore critical to effective collaboration between stakeholders.

The current study examines how design intent expressed through various design representations (Goldschmidt, 1997; Pei et al., 2008) may implicate communication between stakeholder groups (designers, managers, engineers). In particular the current paper reports part of a larger study to understand how the comprehension of design ideas expressed through explorative sketch representations may implicate communication and understanding.

\section{Design representation and communication}

Despite the emergence of participatory or Co-design as means to drive more appropriate design solutions in the face of increasingly complex design problems requiring interdisciplinary approaches (Johnson, 
2005; Karlgren and Ramberg, 2012), the experienced design practitioner's skills in communication through visual representation continue to be acknowledged as a key driver for the synthesis of ideas between stakeholders during conceptual design proposition and development. This is likely because of the ambiguity of the designer's sketch provides rich sources of inspiration, with the potential to open new avenues of exploration (Self, 2017). However, this same ambiguity, important to conceptual design, has the potential to inhibit clear communication and so impact shared understanding, appropriate concept ideation and choice.

Although progress has been made in understanding how sketches and illustrations are used as drivers for design practices (Goel, 1995; Lawson, 2004; Bar-Eli, 2013; Goldschmidt and Rodgers, 2013; Self et al., 2014), less is known of interaction effects between differences in the design representations themselves (Pei et al., 2011) and stakeholder expertise as implicating how they are understood. With the growing complexity of products and product services (Norman, 2011), co-creativity and competitive design-driven innovation (Verganti, 2008) will increasingly be best achieved through shared understanding across a diversity of knowledge, skills and expertise. The current study aims to support shared understanding through the appropriate choice and use of design representations as tools for communication.

When engaging design representations, stakeholder interpretation is the result of an interaction effect between the individual's own foregrounding knowledge and the characteristics of the design representation itself. As such, both the subjective interpretation required in understanding design representations, symptomatic of the undefined nature of conceptual design, together with the characteristics of the representation itself (i.e. idea and study sketches, usability and memory sketches), can influence the communication of design ideas. As Co-design is by definition an activity of shared knowledge and understanding, these same differences implicate how design ideas are actually comprehended by various stakeholders. Thus, the designers' intentions are filtered through stakeholder interpretation to influence the communication of intent, with implications for shared understanding, meaning and preference. However, little is yet known of how the designer's preferred mode of communication (i.e. representational approximations of design intent as drawings, sketches and illustrations) may implicate this communication.

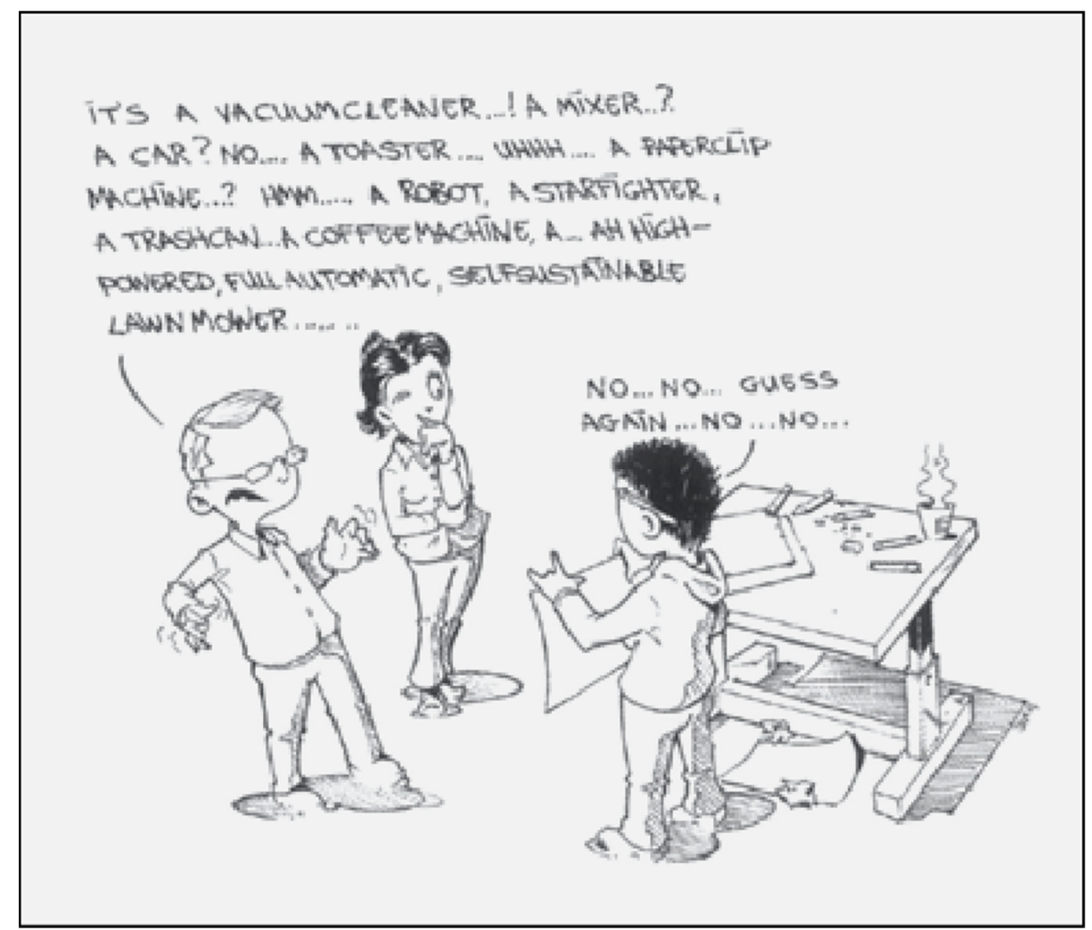

Figure 1. Sketch use in communication with stakeholders (Eissen and Steur, 2010) 


\section{Research aims}

We examined how various design representations implicate communication of design intent. In doing we attempt to address the following research question:

- What is the influence of stakeholder expertise on design communication when expressed through various design representations?

The research question above focuses our analysis on the representations themselves: their purpose of use (i.e. explorative, persuasive, explanatory, and prescriptive), associated characteristics (i.e. ambiguity, detail, fidelity); and how their communication/interpretation is implicated by differences in knowledge and expertise. For, as indicated by Goldschmidt (1991), the meaning of a design representation is constructed, insofar as the subject viewing it interprets its significance.

\section{Methods}

\subsection{Participants}

Following Ulrich and Eppinger's (2012) description of roles and responsibilities within the new product development process, three participant groups were identified (Table 1). Adopting a purposeful sampling approach candidates were contacted and invited to participate. Table 1 describes the participants' professional profiles, including role within a process of new product development.

Table 1. Professional characteristics of participants $(\mathrm{N}=45)$

\begin{tabular}{|c|c|c|c|c|}
\hline \multirow[t]{2}{*}{ Variable } & $\begin{array}{c}\text { Manager } \\
\text { (marketing) } \\
(\mathrm{N}=15)\end{array}$ & $\begin{array}{c}\text { Engineer } \\
(\mathrm{N}=15)\end{array}$ & $\begin{array}{c}\text { Designer } \\
(\mathrm{N}=15)\end{array}$ & Total \\
\hline & $\mathrm{n}$ & $\mathrm{n}$ & $\mathrm{n}$ & $\mathrm{n}$ \\
\hline \multicolumn{5}{|c|}{ Core function description } \\
\hline $\begin{array}{l}\text { Lead role in defining the design to best } \\
\text { meet user needs including aesthetics, } \\
\text { ergonomics, user interface. }\end{array}$ & - & - & 15 & 15 \\
\hline $\begin{array}{l}\text { Plays lead role in mechanical, } \\
\text { electronic design etc. Also responsible } \\
\text { for production system. }\end{array}$ & - & 15 & - & 15 \\
\hline $\begin{array}{l}\text { Mediates interactions between firm \& } \\
\text { consumer. Facilities definition of } \\
\text { market. Oversees product launch. }\end{array}$ & 15 & - & - & 15 \\
\hline \multicolumn{5}{|c|}{ Discipline Experience at time of study (years) } \\
\hline $01-02$ & 6 & 6 & 4 & 16 \\
\hline 03-04 & 6 & 2 & 3 & 11 \\
\hline $05-06$ & 2 & 3 & 4 & 9 \\
\hline $07-10$ & 1 & 3 & 2 & 6 \\
\hline $11+$ & - & 1 & 2 & 3 \\
\hline \multicolumn{5}{|c|}{ Highest educational level } \\
\hline Undergraduate degree & - & 3 & 2 & 5 \\
\hline Masters degree & 13 & 7 & 6 & 26 \\
\hline Ph.D. Degree & 2 & 5 & 7 & 14 \\
\hline
\end{tabular}

\subsection{Design representation as stimuli}

An adapted version of a taxonomy of design representations described by Pei et al. (2011) and extended by Kim et al. (2013) was employed as means to identify and describe different design representations often used during new product development (Figure 2). 


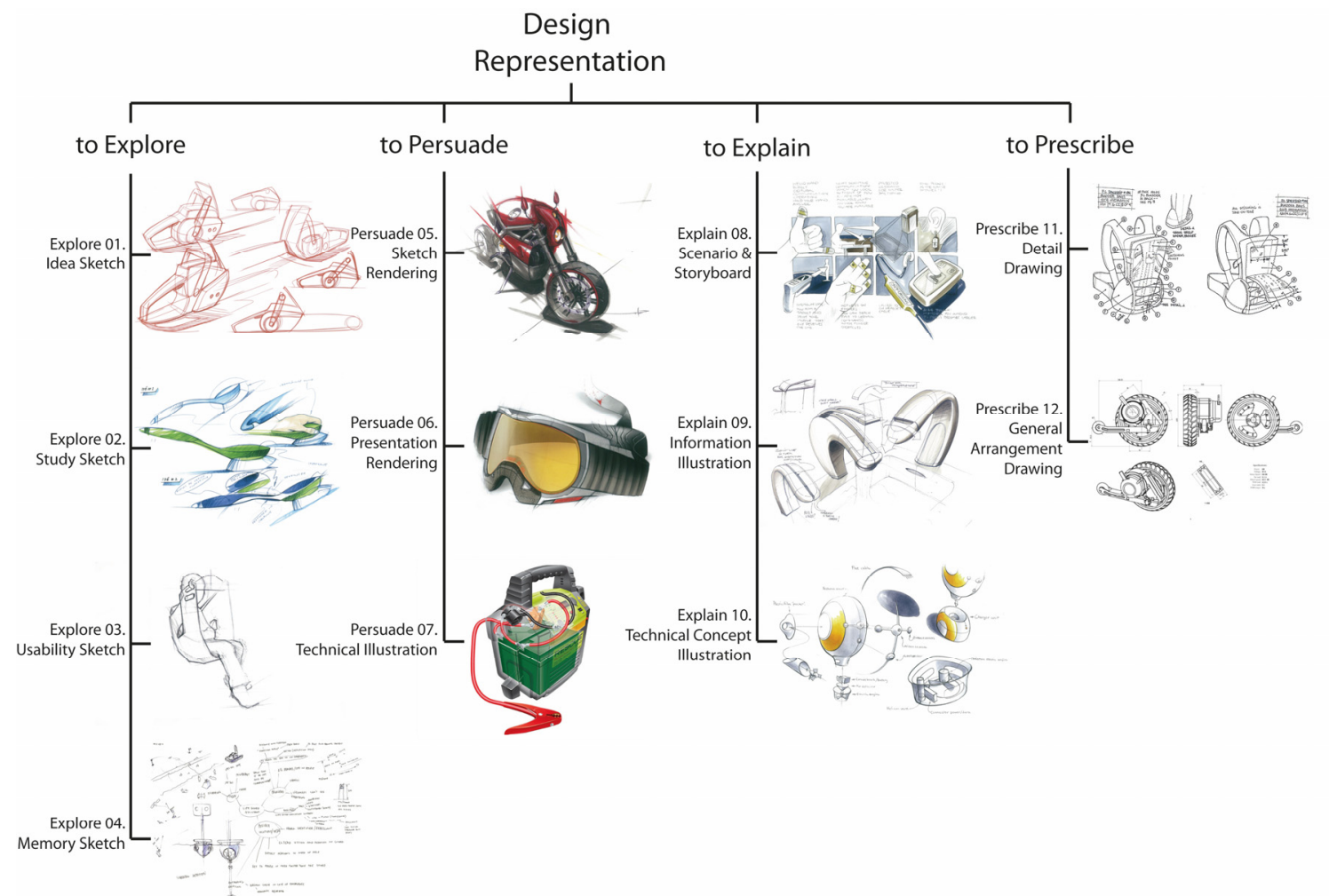

Figure 2. Taxonomy of design representation used as stimuli (Pei et al., 2011; Kim et al., 2013)

12 design representations were classified within four sub-categories according to their purpose of use (Ferguson, 1992). In summary, Representations to Explore supports the exploration of initial design ideas and stimulate first intentions towards the loose evaluation of possible solutions. Representations to Persuade are described as communicating intentions towards product form, design language and aesthetics and are used as means to convince stakeholders of a design's potential (Eissen and Steur, 2010). Explanatory representations provide opportunities to communicate still relatively conceptual ideas in more detail and at a higher level of fidelity (Jonson, 2002), while maintaining some ambiguity to allow interpretation and change (Goel, 1995). Prescriptive representations and illustrations communicate clearly defined detail, often adopting prescribed and systematic methods of representation (i.e. General Arrangement Drawing, Figure 2). Due to space and time constraints, and the current paper's focus upon a conceptual design phase in new product development, the current study reports on an analysis of participant response to the first, explorative category of design representation (Figure 2, Design Representation to Explore).

\subsection{Research procedure}

Eye tracking as a measure of physical response to examine design cognition has recently emerged as an approach to understand design thinking when engaged in conceptual ideation (Sun et al., 2014); although the approach is yet to be employed to examine differences in stakeholder comprehension of design ideas when communicated through concept sketches.

The 12 design representations (Figure 2) were used as stimuli for an eye-tracking study of physiological responses. These responses were then used as data to derive differences between the three stakeholder groups (Table 1, designers, engineers, managers) in how they saw and understood the designs as expressed and communicated through the 12 representation types (the current paper report on the first 4, explorative representations depicted in Figure 2).

Each session was conducted with a single participant seated in front of a wide-screen display at a distance of approximately $40 \mathrm{~cm}$ from the screen. Light screening boards were placed around the desk 
and seat area within a lab environment to limit the effect of lighting upon physiological response (Figure 3). A Tobii X2-60 (Tobii, 2018) stand-alone eye tracker mounted onto a HD monitor was used in combination with Tobii software to gather participant response data on a number of dimensions (i.e. fixation durations, frequencies of fixation, gaze positions, gaze path and pupil diameter). However, the current paper reports results for gaze point fixations (attention) and pupil diameter (cognitive workload). For each session, the 12 design representations were presented to the participant in random order to limit order effect. Each representation was on screen for 15 seconds, interspaced with prompt slides appearing for 5 seconds each. Participants were advised before each session to remain still and to look at the screen until told by the researcher to do otherwise. Each eye-tracking session lasted for approximately four minutes.
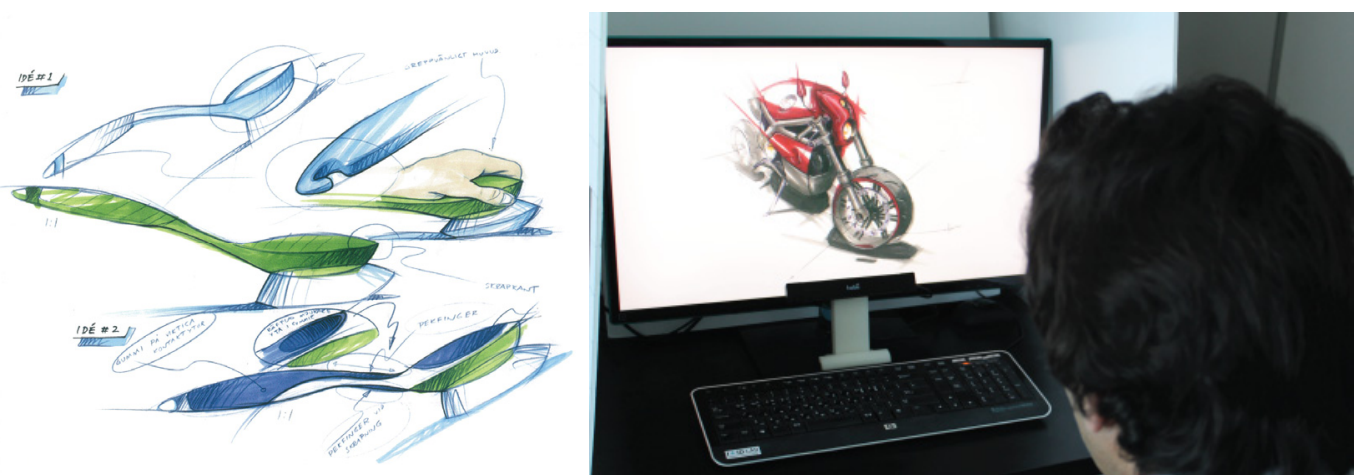

Figure 3. Design representation stimuli image (left) and eye-tracking session (right)

Upon completion of the eye-tracking session, participants participated in a card-sorting exercise and open-ended interview session (not reported in the current paper). At the end of each session, all resulting eye-tracking data was archived using participant codes for later analysis. Figure 4 illustrates an example gaze track (left) and heat map (right) generated from an eye-tracking study session.
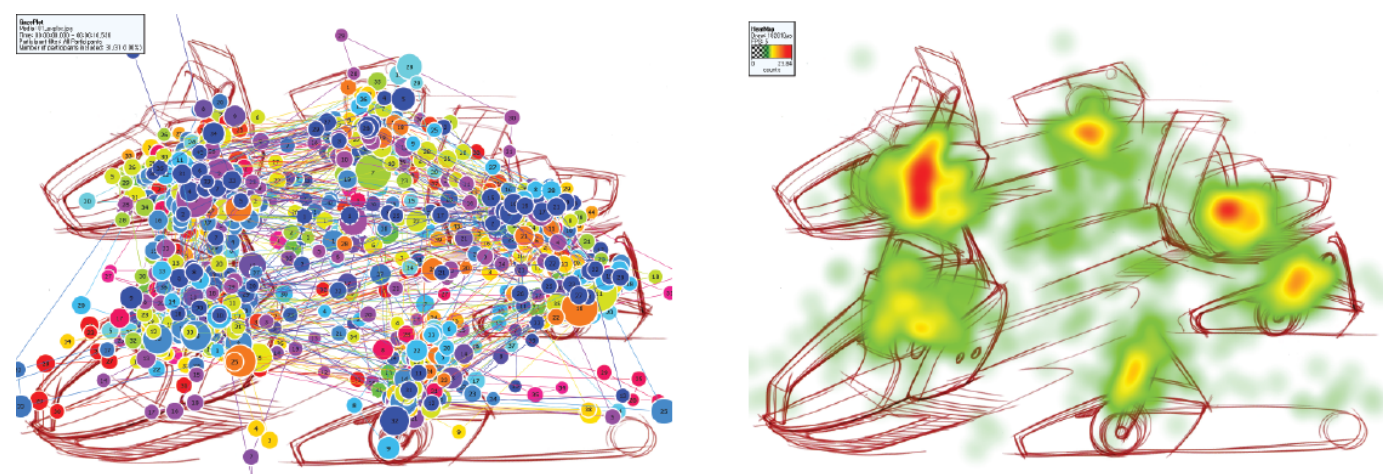

Figure 4. Visualisation of gaze-track (left) and heat map (right) data

\section{Results}

In order to understand how the various representations illustrated in Figure 2 above may be comprehended differently by the three stakeholder groups (designers, managers, engineers), together with potential implications for communication, we analysed eye-tracking data for gaze point fixations (qualitative) and pupil dilation (quantitative). To achieve this we produced heat-map images based upon gaze point fixations to illustrate differences in attention between the participant groups. We then compared fixations with the subjects' pupil dilations to statistical analysis (One-way ANOVA) differences in dilation between participant groups, indicating cognitive load (i.e. comprehendability). In this way we explored physiological responses as indicators for interest (attention through gaze-point fixations) and understanding (cognitive load through pupil dilation). The current paper reports results for design stimuli representations 01 to 04 (to Explore representations, Figure 2). 


\subsection{Explore 01.Idea Sketch}

Figure 5 illustrates the stimuli Explore 01.Idea Sketch. The sketch type expresses solution ideas at a low level of detail and fidelity. The idea sketch is described as quickly externalising thoughts using simple line-work and is most often used to communicate between designers or for personal use by a single designer (Pei et al., 2011).

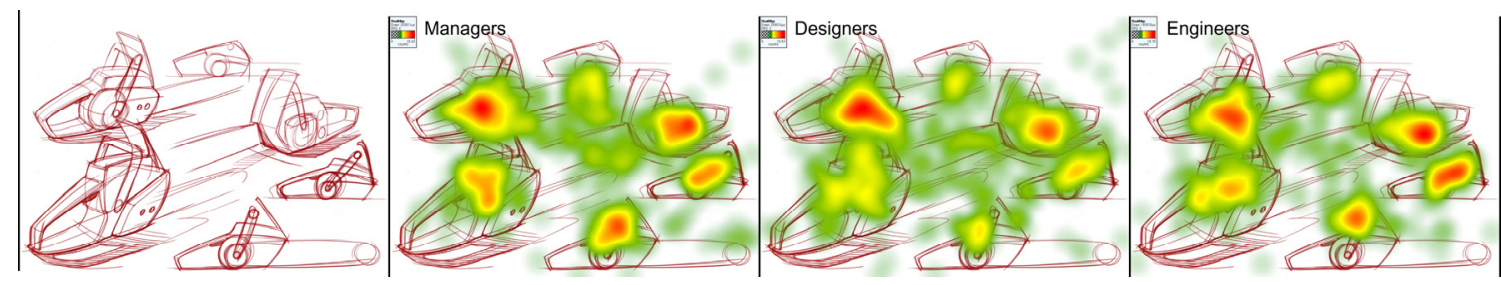

Figure 5. Explore 01.Idea Sketch, heat mapping between participant groups

As indicated in Figure 5, visual inspection of heat mapping indicated some difference in attention between stakeholder groups. For example, the designers did not appear to pay as much attention to the handle details of the chain-saw design (Figure 5, bottom left and right fixations). This may have indicated the engineers and managers were more attentive to/interested in use and technical detailing compared to the designer participants. Likewise the designers attention appeared more widely distributed across the image, indicated by fewer red gaze point fixations $(f=3)$ compared to both managers $(f=5)$ and engineers $(f=5)$. This may also have indicated the designers' tendency to more holistically take in an overview of the image compared to the other two stakeholder groups. If this was the case, it appeared explorative idea sketches may communicate to designers a flavour of potential solution ideas. In contrast, engineers and managers, even when ideas are communicated at the low-levels of detail and fidelity offered by the explorative Idea Sketch, may try to understand the detail of the design intent. If so, consideration for this difference is required when ideas are communicated through more explorative and ambiguous representations, as engineers and managers may attempt to interpret idea sketches in terms their more literal ability to describe and explain a design.

In terms pupil dilations (indicating cognitive workload in comprehension), data related to Explore01 Idea Sketch was lost, and thus we are unable to report these results here.

\subsection{Explore 02.Study Sketch}

Figure 6 shows the Explore 02.Study Sketch stimuli, described as used to explore the detail of potential solution ideas at a higher level of fidelity compared to Explore01.Idea Sketch (Olofsson and Sjolen, 2005). The sketch illustrated in Figure 6 is achieved through line-work accompanied by colour rendering and some notational annotation.

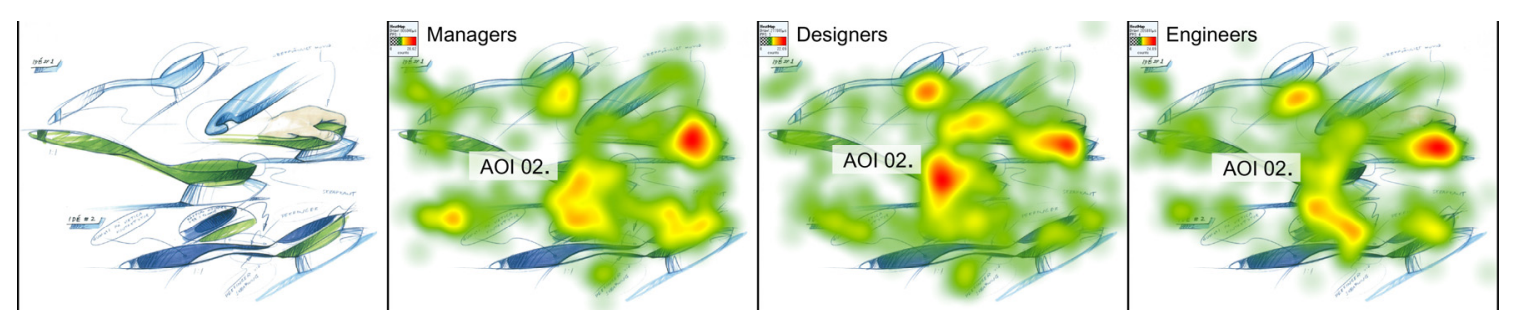

Figure 6. Explore 02.Study Sketch, heat mapping between participant groups

As indicated by Figure 6, the designers appeared to give the dominant rendering in the centre of the Study Sketch stimuli greater attention compared to the engineers and managers (Figure 6, centre red gaze point fixation, AOI (Area of Interest) 02). Likewise, although all three groups appeared to attend to the human hand image (upper-right), the designers attention was drawn more so to its handle detail. In the case of the former, it may have been that the designers attended to the more dominant, visual image compared to the managers, and engineers. The designers' attention to the handle design may have also indicated their interest in the visual aspects of the stimuli. As with Explore 01.Idea Sketch (above), 
the designers appeared to give overall attention to the Study Sketch stimuli compared to both engineers and managers, indicated by reduced colouration of the image and fewer red/yellow gaze point fixations (Figure 6). This result may have suggested how designers' attention was both drawn more to the visual elements of the Study Sketch. In contrast the engineers may have looked for cues to aid understanding of how the potential solution may work in terms use and technical functionality.

A statistical analysis of pupil dilation was also performed (One-way ANOVA) on pre-defined areas of interest (AOI) to indicate cognitive load (difficulty and comprehendability) in understanding design as communicated by the Explore 02.Study Sketch. Results showed significant difference between the participant groups in terms the dominant centre image (Figure 6, AOI 2). Tables 2 and 3 provide the descriptive and statistical results.

Table 2. Explore 02.Study Sketch, AOI 02, descriptive statistics

\begin{tabular}{ccccc}
\hline Subject & Count & Sum & Mean & Variance \\
\hline Managers & 261 & 770.925 & 2.953735632 & 0.138835 \\
Designers & 365 & 981.435 & 2.688863014 & 0.086719 \\
Engineers & 400 & 1125.675 & 2.8141875 & 0.109516 \\
\hline
\end{tabular}

Table 3. Explore 02.Study Sketch, AOI 02, ANOVA result

\begin{tabular}{ccccccc}
\hline Source & Square Sum & DOF & Square Mean & F Ratio & p-value & $\begin{array}{c}\text { F critical } \\
\text { value }\end{array}$ \\
\hline Factor & 10.73071741 & 2 & 5.365358704 & 49.28859 & 0.00 & 3.004522066 \\
Residual & 111.3596968 & 1023 & 0.108856009 & & & \\
Total & 122.0904143 & 1025 & & & & \\
\hline
\end{tabular}

As indicated in Table 2, the designers mean pupil dilation $(\bar{x}=2.69)$ was lower than both managers $(\bar{x}=2.95)$ and engineers $(\bar{x}=2.81)$. This result would indicate the managers and engineers were working harder than the designers (cognitive workload) in their attempts to understand AOI 02. This result was confirmed to be statistically significant (Table 3 ). If this was the case, the result indicated managers and engineers may find designs communicated through explorative study sketches more challenging to comprehend compared to designers. We speculate that this result, indicative of challenge in understanding, may have lead to the engineers increased attention to detail (indicated by fewer gaze point fixations, Figure 6). In contrast designers may be more inclined to see Study Sketches more holistically thus requiring less workload to reading them.

\subsection{Explore 03.Usability Sketch}

Figure 7 illustrates a representation type used to express intent towards usability within a context of use (Kim et al., 2013). In the case of Figure 7, the illustration of a human form as expression of intended use produced using pencil line-work with indication of light and shadow through shading.

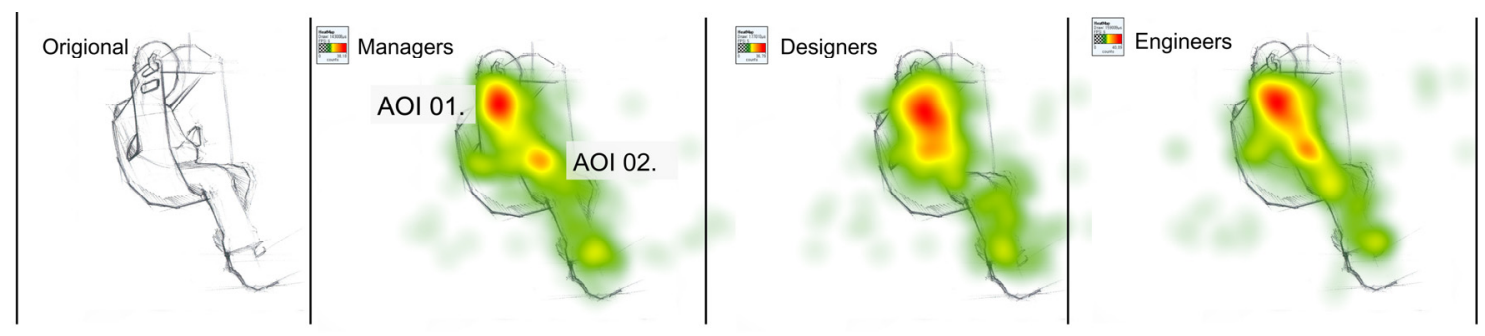

Figure 7. Explore 03.Usability Sketch, heat mapping between participant groups

The qualitative visualisation of gaze point fixations (Figure 7), indicated the managers as appearing to split interest between the illustrated user's head and legs (Figure 7, two distinct fixation points, AOI 01 and AOI 02). This was in contrast to the designers and engineers closer fixations between AOI 01 and 
AOI 02 . The result above also appeared to relate to a statistically significant difference in pupil dilations for AOI 02 (Figure 7) between the three participant groups (Tables 4 and 5).

Table 4. Explore 03.Usability Sketch, AOI 02, descriptive statistics

\begin{tabular}{ccccc}
\hline Subject & Count & Sum & Mean & Variance \\
\hline Managers & 528 & 1458.015 & 2.761392045 & 0.118434 \\
Designers & 431 & 1103.645 & 2.560661253 & 0.085267 \\
Engineers & 661 & 1755.4 & 2.655673222 & 0.086357 \\
\hline
\end{tabular}

Table 5. Explore 03.Usability Sketch, AOI 02, ANOVA result

\begin{tabular}{ccccccc}
\hline Source & Square Sum & DOF & Square Mean & F Ratio & p-value & F critical value \\
\hline Factor & 9.655450639 & 2 & 4.827725319 & 50.01718 & 0.00 & 3.001289173 \\
Residual & 156.0750138 & 1617 & 0.096521344 & & & \\
Total & 165.7304644 & 1619 & & & & \\
\hline
\end{tabular}

As indicated in Table 4, the designers pupil dilation was reduced $(\overline{\mathrm{x}}=2.56)$, compared to both managers $(\overline{\mathrm{x}}=2.76)$ and engineers $(\overline{\mathrm{x}}=2.66)$. This result again indicated (see also Explore 02.Study Sketch above) the designers found engaging with the AOI of the Usability Sketch less challenging (cognitive workload), compared to the other two participant groups. If this result holds for the usability sketch in its entirety it would suggest designers find the sketch type easier to comprehend compared to managers and engineers. Although in terms attention, all three groups appeared drawn to the human form within the Usability Sketch stimuli.

\subsection{Explore 04.Memory Sketch}

Figure 8 shows stimuli Explore 04.Memory Sketch, described as used to expand ideas during the design process through mind maps, notes and annotations (Pei et al., 2011). The Memory Sketch chosen was produced using black fine-line pen work, accompanied by grey marker rendering.
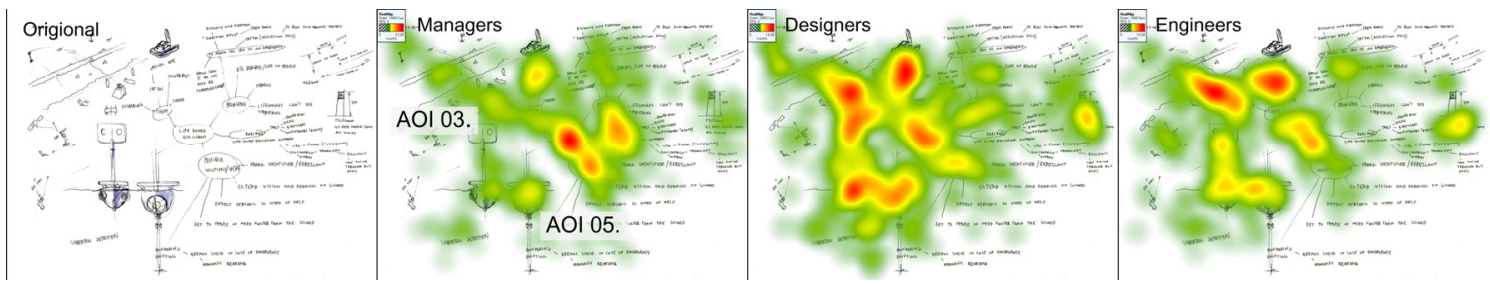

Figure 8. Explore 04.Memory Sketch, heat mapping between participant groups

As indicted in Figure 8, the designers showed increased gaze point fixations (Figure 8, red heat areas, $f=6$ ), compared to both managers $(f=4)$ and engineers $(f=4)$. In particular the designers' gaze point fixations concentrate upon the Memory Sketch's visual elements, when compared to the managers and engineers. Again, this result may have indicated (see also Explore 02.Study Sketch above) how the designers attention is drawn to the visual elements of the sketch stimuli. In contrast, the managers in particular appeared to attend more to the systemic diagrammatic elements of the Memory Sketch. If this is the case, it would indicate how managers may be more inclined towards diagrammatic elements compared to the other two groups. That is, they may attend more greatly to and/or be stimulated by systemic considerations and their relationships.

Perhaps related to this result, a statistical analysis of pupil dilation (cognitive workload indicative of comprehension) indicated significant difference between the groups in terms two AOIs (Figure 8, AOI 3 and AOI 5, Tables 6 to 9). 
Table 6. Explore 04.Memory Sketch, AOI 03, descriptive statistics

\begin{tabular}{ccccc}
\hline Subject & Count & Sum & Mean & Variance \\
\hline Managers & 187 & 507.785 & 2.715427807 & 0.031316 \\
Designers & 396 & 1017.2 & 2.568686869 & 0.037041 \\
Engineers & 824 & 2216.31 & 2.689696602 & 0.047522 \\
\hline
\end{tabular}

Table 7. Explore 04.Memory Sketch, AOI 03, ANOVA result

\begin{tabular}{ccccccc}
\hline Source & Square Sum & DOF & $\begin{array}{c}\text { Square } \\
\text { Mean }\end{array}$ & F Ratio & p-value & $\begin{array}{c}\text { F critical } \\
\text { value }\end{array}$ \\
\hline Factor & 4.601820123 & 2 & 2.300910062 & 54.23271 & 0.00 & 3.002133407 \\
Residual & 59.5669571 & 1404 & 0.042426608 & & & \\
Total & 64.16877722 & 1406 & & & & \\
\hline
\end{tabular}

Table 8. Explore 04.Memory Sketch, AOI 05, descriptive statistics

\begin{tabular}{ccccc}
\hline Subject & Count & Sum & Mean & Variance \\
\hline Manager & 102 & 266.79 & 2.615588235 & 0.142024 \\
Designer & 252 & 636.06 & 2.524047619 & 0.026347 \\
Engineer & 152 & 383.165 & 2.520822368 & 0.071688 \\
\hline
\end{tabular}

Table 9. Explore 04.Memory Sketch, AOI 05, ANOVA result

\begin{tabular}{ccccccc}
\hline Source & Square Sum & DOF & Square Mean & F Ratio & p-value & $\begin{array}{c}\text { F critical } \\
\text { value }\end{array}$ \\
\hline Factor & 0.701629656 & 2 & 0.350814828 & 5.552106 & 0.004120794 & 3.013645099 \\
Residual & 31.78250834 & 503 & 0.063185901 & & & \\
Total & 32.48413799 & 505 & & & & \\
\hline
\end{tabular}

As indicated in Tables 6 and 8, the designers mean pupil dilation for AOI $03(\bar{x}=2.57)$ and AOI 05 $(\bar{x}=2.52)$ was reduced compared to the other two groups. In terms AOI 03, the engineers received the higher fixation count (Table 6, $f=824$ ), compared to designer (Table $6, f=396$ ) and managers $(f=187$ ). This would indicate the engineers as having most interest in AOI 03, but that the managers where most challenged to comprehend it (pupil dilation, $\bar{x}=2.7$ ). As AOI 03 communicates a point of detail as a figurative thumb-nail image, it may be that the designers and engineers paid it significantly more attention. If this is the case it is evidence to suggest how designer and engineers may be more attentive towards the visual elements of a Memory Sketch (Figure 8, AOI 03 and 05, red gaze point fixations). In contrast, the managers appeared more concerned with its diagrammatic elements (Figure 8, gaze point, fixations towards right of image (systemic-diagram), managers, and lack thereof for engineers). This result was supported by the gaze point heat mapping for AOI 05 (Figure 8). The designers' heat map indicating increased gaze point attention (in red) compared to less for the managers. This result was supported by fixation counts for AOI 05 between the three groups (Table 8 , designer $f=252$, engineer $f=152$ and manager $f=102$ ). Thus, although increased designer gaze point fixation was identified at AOI 05 of Explore 04.Memory Sketch, the designers' pupil dilation indicated reduced cognitive load in understanding this, more visual, area of the sketch stimuli $(\bar{x}=2.52)$. That is they were both more attentive to the visual stimuli, but at the same time more comfortable in their interpretation of the design representation.

\section{Discussion and conclusions}

The current paper has presented a section of a larger study aimed at understanding the influence of stakeholder expertise on design communication when expressed through various design representations. 
To achieve this research aim we recruited a sample of stakeholders from the various disciplines (designers, managers, engineers) represented within a process of new product development (NPD) (Ulrich and Eppinger, 2012). Through literature review, and the author's existing works related to design representation (Ferguson, 1992; Olofsson and Sjolen, 2005; Pei et al., 2008, 2011; Kim et al., 2013) we identified and positioned a taxonomy of design representations. The current study reports comparative results related to participant responses towards explorative design representations (Explore 01.Idea Sketch, Explore 02.Study Sketch, Explore 03.Usability Sketch, Explore 04.Memory Sketch). These representation types are described as most often used during design ideation at the front end of new product development to identify, explore and communicate potential conceptual solution ideas. This stage in the process has been described as both critical to the success of new product development and requiring careful collaboration and communication between different stakeholders to provide the most opportune conditions for success (Nikander et al., 2014).

With the design representations as stimuli, the stakeholders (designers, managers, engineers) participated in an eye-tracking study to collect physiological response data indicating their initial response to the sketch stimuli. Specifically, we gathered data on attention (gaze point fixations) and comprehension (pupil dilation). Adopting a mixed qualitative/quantitative approach to data analysis, gaze point fixations where illustrated through heat mapping to indicate attention. These results were supported by a statistical analysis (One-way ANOVA) of pupil dilation to explore differences in cognitive workload. These results we interpreted as degree of difficulty in comprehensibility. Within the boundaries of the current paper we provide some initial findings.

First, results indicated how designers may be more inclined to holistically comprehend design when represented through conceptual sketches and illustrations. For example, gaze point fixations seen in Explore 01. Idea Sketch indicated the designers' attention was increasingly concerned with the representation as a whole, rather than fixating on particular areas of the stimuli. Likewise, gaze point fixation heat mapping for stimuli Explore 02.Study Sketch indicated the designers' attention towards the various areas of the sketch, in contrast to the managers and engineers close attention to specific details within the stimuli. In contrast, increased gaze point fixations at specific areas of Explore 01. Idea Sketch may have been indicative of the engineers and managers attention to the detail of design ideas when expressed as conceptual sketches and illustrations. If this is the case, careful consideration is required in communication of design intent through these explorative design representations because engineers and managers may be inclined to attempt to understand the designs they express as more literal representations of the intended design outcome, and so attempt to understand them within this frame of reference through focus on detail. However, as the purpose of explorative sketches is to provide a flavour of the potential solution idea, the managers and engineers focus on understanding may have resulted in increased cognitive workload. That is, there frame of reference may have implicated challenge in comprehension indicated through increased workload. On the other hand, the designers may comprehend the sketch representation as expressing a potential design idea as open to further suggestion and refinement, with evidence of this identified as reduced pupil dilation (cognitive load). Related to the above, results indicated how stakeholder expertise may have implicated the kinds of design details attended to within the sketch stimuli. For example, increased designer gaze point fixation was identified at the area of Explore 04.Memory Sketch, depicting a visual expression of design intent (AOI 05, Memory Sketch). In contrast the managers' attention was drawn towards diagrammatic elements of the same representation, indicated by increased gaze point fixation upon systemic, diagrammatical elements of the Memory Sketch. It may be that the managers were inclined to pay more attention to systemic considerations, compared to the designers. If this is the case the result indicated how different stakeholders attribute more or less importance to the representation of the visual/aesthetic/form elements of a proposed design solution compared to consideration of systemic, technical or contextual aspect. In terms the Memory Sketch representation, these differences appeared to depend upon stakeholder expertise, with the managers' fixation on diagrammatic, systemic elements of the representation, compared to the designers focus on visual, product depictions.

Third, results indicated how engineers and managers may be more challenged in comprehending design ideas when communicated through sketch representations compared to the designers. For example, results of pupil dilation for the engineers, and in particular the managers, when presented with Explore 
02.Study Sketch indicated increased cognitive workload in comprehending the sketch stimuli. In contrast physiological responses obtained from the designers indicated reduced challenge in comprehension. Likewise, the designers appeared less challenged in comprehending the illustrative, visual elements of design representation Explore 04.Memory Sketch, as indicated by reduced pupil dilation, whereas the managers and engineers appeared to spend more time attempting to attend to details. It may be that the designers comprehended the sketch representation as more illustrative of potential solution ideas, and were also more comfortable with this interpretation. In contrast, the engineers and managers may have interpreted the sketch representations as more concrete in the proposition of ideas, and in doing, sort evidence of more specific detail. This in turn may have resulted in increased cognitive load.

However, these observation remain speculative in that more work is required to better understand how stakeholder expertise may implicate comprehension (and ultimately communication) of ideas during new product development. For example, the current study did not account for the influence of context upon communication. For example, how might communication be implicated when design representations are accompanied by oral explanation and/or gestural indicators (Zurita et al., 2008). Moreover, although the collection of physiological responses through eye-tracking can provide indicators of attention and comprehendability, other studies are required to support and/or refute our own results. For example, interviews may be conducted to understand stakeholders' experiences of communication through design representation during new product development. Although the current work provides evidence to suggest the stimulation of indicators for increased attention and cognitive loading, our approach does little to understand the potential implications for actual communication of intent. Observational and self-report methods may help to uncover relationships between our results and communication through conceptual sketches and illustrations.

These limitations notwithstanding, the results presented in this study provide a point of departure for further work to understand the role and importance of design representations as they are, and continue to be used as a critical means of communication to support creative product development.

\section{Acknowledgements}

This work was supported by the Ministry of Education of the Republic of Korea and the National Research Foundation of Korea (NRF-2015S1A5A8015329). We would like to thank Geehyuck Jeong and Seong Keun Lee for their research support to this project.

\section{References}

Bar-Eli, S. (2013), "Sketching profiles: Awareness to individual differences in sketching as a means of enhancing design solution development", Design Studies, Vol. 34 No. 4, pp. 472-493. https://doi.org/10.1016/j.destud.2013.01.007

Eissen, K. and Steur, R. (2010), Sketching: Drawing techniques for product designers, BIS Publishers, Amsterdam.

Ferguson, E.S. (1992), Engineering and the Mind's Eye, MIT Press, Cambridge.

Goel, V. (1995), Sketches of Thought, MIT Press, London.

Goldschmidt, G. (1997), "Capturing indeterminism: representation in the design problem space", Design Studies, Vol. 18 No. 4, pp. 441-455.

Goldschmidt, G. (1991), “The dialectics of sketching”, Creativity Research Journal, Vol. 4 No. 2, pp. 123-143. https://doi.org/10.1080/10400419109534381

Goldschmidt, G. and Rodgers, P.A. (2013), "The design thinking approaches of three different groups of designers based on self-reports", Design Studies, Vol. 34 No. 4, pp. 454-471. https://doi.org/10.1016/j.destud.2013.01.004

Holtzblatt, K., Wendell, J. and Wood, S. (2005), Rapid Contextual Design: A how-to guide to key techniques for user-centred design, Morgan Kaufmann Publishers, London.

Johnson, J. (2005), "Complexity science in collaborative design", CoDesign, Vol. 1 No. 4, pp. $223-242$. https://doi.org/10.1080/15710880500478346

Jonson, B. (2002), "Sketching Now", The International Journal of Art and Design Education, Vol. 21 No. 3, pp. 246-253.

Karlgren, K. and Ramberg, R. (2012), "The use of design patterns in overcoming misunderstandings in collaborative interaction design", CoDesign, Vol. 8 No. 4, pp. 231-246. https://doi.org/10.1080/15710882.2012.734829 
Kim, S., Jung, S. and Self, J. (2013), "Investigating Design Representation: Implications for an Understanding of Design Practice", IASDR13 Consilience and Innovation in Design, Tokyo.

Lawson, B. (2004), What Designers Know, Architectural Press, Oxford.

Nikander, J.B., Liikkanen, L.A. and Laakso, M. (2014), "The preference effect in design concept evaluation", Design Studies, Vol. 35 No. 5, pp. 473-499. https://doi.org/10.1016/j.destud.2014.02.006

Norman, D. (2011), Managing Complexity A Partnership Living with Complexity, The MIT Press, Cambridge.

Olofsson, E. and Sjolen, K. (2005), Design Sketching, KEEOS Design Books AB, Sweden.

Pei, E., Campbell, R. and Evans, M. (2008), "Building a Common Ground: The Use of Design Representation Cards for Enhancing Collaboration between Industrial Designers and Engineering Designers", DRS2008 Undisciplined, Sheffield, UK.

Pei, E., Evans, M. and Campbell, I. (2011), “A Taxonomic Classification of Visual Design Representations Used by Industrial Designers and Engineering Designers", The Design Journal, Vol. 14 No. 1, pp. 64-91. https://doi.org/10.2752/175630610X12877385838803

Sanders, E.B.N. and Stappers, P.J. (2008), "Co-creation and the new landscapes of design”, CoDesign, Vol. 4 No. 1, pp. 5-18. https://doi.org/10.1080/15710880701875068

Self, J. (2017), "Resolving Wicked Problems: Appositional Reasoning and Sketch Representation", The Design Journal, Vol. 20 No. 3, pp. 313-331. https://doi.org/10.1080/14606925.2017.1301070

Self, J., Evans, M. and Dalke, H. (2014), "Design Activity Perceptions and Performance: Investigating the relationship between expertise and practice", The Design Journal, Vol. 17 No. 3.

Sun, L., Xiang, W. and Yang, C. (2014), "The Role of Sketching States in the Stimulation of idea Generation: An Eye movement Study”, Creativity Research Journal, Vol. 26 No. 3, pp. 305-313.

Tobii (2018), Tobii Group Inc. [online] Tobii. Available at: https://www.tobii.com/group/ (accessed 10.03.2018).

Ulrich, K. and Eppinger, E. (2012), Product Design and Development, 5th ed., McGraw-Hill Education, New York.

Verganti, R. (2008), "Design, Meanings, and Radical Innovation: A Metamodel and a Research Agenda*”, Journal of Product Innovation Management, Vol. 25 No. 5, pp. 436-456. https://doi.org/10.1111/j.15405885.2008.00313.x

Zurita, G., Baloian, N. and Baytelman, F. (2008), “A collaborative face-to-face design support system based on sketching and gesturing", Advanced Engineering Informatics, Vol. 22 No. 3, pp. 340-349. https://doi.org/10.1016/j.aei.2007.09.003

Prof. James Andrew Self, Associate Professor of Industrial Design

UNIST (Ulsan National Institute of Science and Technology), Industrial Design

50 UNIST-gil, Ulju-gun, 689-798 Ulsan, South Korea

Email: jaself@unist.ac.kr 